\title{
Not Minding the Gap: Does Ride-Hailing Serve Transit Deserts?
}

\author{
Jesus M. Barajas \\ University of California, Davis \\ jmbarajas@ucdavis.edu \\ Anne Brown \\ University of Oregon \\ abrown33@uoregon.edu
}

November 18, 2020

\begin{abstract}
Transit has long connected people to opportunities but access to transit varies greatly across space. In some cases, unevenly distributed transit supply creates gaps in service that impede travelers' abilities to cross space and access jobs or other opportunities. With the advent of ride-hailing services like Uber and Lyft, however, travelers now have a new potential to gain automobility without high car purchase costs and in the absence of reliable transit service. Research remains mixed on whether ride-hailing serves as a modal complement or substitute to transit or whether ride-hailing fills transit service needs gaps. This study measures transit supply in Chicago and compares it to ride-hailing origins and destinations to examine if ridehailing fills existing transit service gaps. Findings reveal clustering of ride-hailing pickups and drop-offs across the City of Chicago, but that the number of ride-hailing pickups and drop-offs was most strongly associated with high neighborhood median household income rather than measures of transit supply. In bivariate analyses, transit service was not associated with ridehailing trip ends. But after controlling for neighborhood socioeconomic status, transit dependency, population density, and employment density, we found fewer ride-hailing trips in neighborhoods where bus service dominated and significantly more ride-hailing trips where rail service was prevalent. Patterns were slightly different for overnight weekend ride-hailing pick-ups, where higher transit density predicted a greater number of trips in nearby tracts. Additional research and policy is needed to ensure that ride-hailing services provide travel options to those who need them the most and fill transit gaps in low-income communities when options to increase service are limited.
\end{abstract}

Keywords: public transit; transit service needs gaps; equity; ride-hail; transportation network company

This is the author's accepted manuscript of an article published in the Journal of Transport Geography, available at https://doi.org/10.1016/j.jtrangeo.2020.102918. This manuscript version is made available under the CC-BY-NC-ND 4.0 license http://creativecommons.org/licenses/by-nc-nd/4.0/ 


\section{Introduction}

Transit provides critical mobility that connects people to opportunities. Access to transit, however, varies greatly across space. Where transit supply is limited, people who depend on transit may have reduced access to jobs, healthcare, or education. And because lowerincome people of color are more likely to ride transit, lack of service presents issues of equity and justice in transportation planning. New technologies, however, may offer an opportunity for travelers to overcome existing transit network gaps. Ride-hailing services like Uber and Lyft-also known as transportation network companies (TNCs) - present a way that travelers can now cross space in the absence of reliable transit service. But potential links between ride-hailing trip-making and existing transit service remain unclear on the supply side, while on the demand side, surveys of ride-hailing users present murky answers to whether ride-hailing is a modal complement or substitute to transit.

In this study, we examine the potential connections between transit service and ride-hailing trip-making in two related research questions. First, where are the gaps in transit service in the City of Chicago and how are they distributed with respect to neighborhood sociodemographic characteristics? And second, what is the spatial connection between transit service gaps, ride-hailing origins and destinations, and neighborhood composition? We answer these questions using transit service and ridehailing trip-making data from the City of Chicago from November 2018 to December 2019. We find evidence that, at a neighborhood scale, ride-hailing does not primarily serve to fill transit service gaps, but instead coexists with transit service as either a complementary or competing mode.

We divide the remainder of this paper into four sections. We first review existing research on transit supply gaps and the potential role that ride-hailing may play in bridging these gaps. We then discuss the data and methods employed in this research. Third, we discuss findings about transit service gaps in the City of Chicago, patterns of ride-hailing trip making, and the associations between them. We conclude with implications for transportation planning and policy.

\section{Background}

\subsection{Uneven access to transit}

Providing transit service has been a prominent transportation goal in the United States since the 1970s (Taylor and Morris, 2015). Transit provides a range of opportunity benefits such as increased rates of labor participation (Sanchez, 1999) and access to healthy foods (Widener et al., 2015). Transit availability, however, which some have used to explain why the poor live in cities (Glaeser et al., 2008), is highly uneven across space. Research shows that transit service and use remain highly concentrated in certain neighborhoods and among a relatively small fraction of travelers. For example, in Melbourne, 70 percent of the population lives near just 19 percent of the transit supply 
(Delbosc and Currie, 2011). And in the Los Angeles region, just 17 percent of neighborhoods are home to 45 percent of transit commuters (Manville et al., 2018). Some researchers attribute the uneven distribution of service-and specifically, the provision of commuter-oriented transit service in lower-density, typically higher-income suburbswith efforts to secure popular support for transit spending at the expense of proving more robust service in urban cores and transit-dependent, often lower-income, populations (Taylor and Morris, 2015).

In some cases, uneven distributions of transit supply create transit service needs gaps. Transit service needs gaps-also referred to as "transit deserts"-are typically defined as areas where transit need is high but transit supply is low or non-existent (AI Mamun and Lownes, 2011b, Currie, 2010, Jiao and Dillivan, 2013, Currie, 2004). In such areas, people without cars or reliable transit service may be unable to access jobs or other opportunities. Researchers most commonly measure transit need or disadvantage using socioeconomic factors including race, ethnicity, income, employment status, car availability, age, and disability; less frequently transit need reflects additional factors such as immigrant status, gender, language fluency, temporal isolation, and unlicensed status (Carleton and Porter, 2018). Carleton and Porter (2018) caution, however, that aggregate metrics of transportation disadvantage can mask differing levels of inequality across groups or be heavily influenced by weighting.

As with transit need, researchers measure transit supply in different ways.

Researchers have used the type and frequencies of transit service (El-Geneidy et al., 2014, Welch and Mishra, 2013), proximity to transit stops (Mavoa et al., 2012, Toms and Song, 2016), temporal schedule variations (Fayyaz et al., 2017, Fransen et al., 2015), and other composite measures of transit accessibility constructed from various transit service characteristics. ${ }^{1}$ Because of temporal variation in transit schedules, transit supply gaps may differ across times of day (Fransen et al., 2015).

In some cities, transit service needs gaps vary systematically across population characteristics. For example, people of color, older, student, and lower-income populations receive lower shares of bus service relative to their shares of the population (Wells and Thill, 2012, Jiao and Dillivan, 2013, Al Mamun and Lownes, 2011b, Currie, 2010). Researchers find evidence of transit deserts across multiple American cities (Jiao, 2017, Toms and Song, 2016, Jiao and Dillivan, 2013). Some find that transit deserts are often concentrated in central neighborhoods in low-income neighborhoods (Jiao and Dillivan, 2013, Toms and Song, 2016), while others find that the locations of transit deserts vary by city (Jiao, 2017). Importantly, how researchers define both transit need and transit supply, as discussed above, influences where transit service gaps are found.

\footnotetext{
${ }^{1}$ For examples of transit accessibility measures, see Al Mamun and Lownes (2011a).
} 


\subsection{Is Ride-hailing a complement or substitute to transit?}

One of the greatest challenges with inadequate transit service is that people who depend on transit and who lack reliable access to both cars and transit may be unable to access jobs or other opportunities across a city. But a new potential now exists for people to gain automobility without high car purchase costs and in the absence of reliable transit service: ride-hailing. Ride-hailing services such as Uber and Lyft connect travelers to drivers through smartphone applications. Across the United States, transit agencies are seeking to capitalize on the potential for ride-hailing to complement transit services, specifically to bridge first-last mile gaps to and from transit stations. Schwieterman et al. (2018) find that, among the dozens of transit agency-ride-hailing partnerships across the US, most are "motivated by a desire to improve mobility in areas in which transit options are inadequate or nonexistent, or where the supply of parking is insufficient" (p. 1). The effects of such partnerships at an agency-wide level remain unclear, however, and there exists a lively ongoing debate about whether ride-hailing acts as a complement or substitute to transit service. Two types of data inform this debate: neighborhood analyses of ride-hailing travel, and surveys of individual ride-hailing users.

Neighborhood analyses typically examine ride-hailing use in terms of aggregate trends across neighborhoods with varied built environment and socioeconomic characteristics. Across multiple cities and regions, researchers find that the number of ride-hailing trips is associated with transit density or being within a half-mile of rapid transit (Brown, 2019a, Gehrke, 2020, Sabouri et al., 2020, Lavieri et al., 2018, Feigon and Murphy, 2018, Jiao and Wang, 2020). Research using trip-level and aggregate ride-hailing data suggests complex tradeoffs between transit and ride-hailing, where ride-hailing and transit may be neither a perfect complement nor substitute and where the relationship is oft shaped by local context such as mode, transit service quality, time of day, wait times, and ride-hailing price. Researchers contrastingly find no clear association between peakhour ride-hailing use and longer-term changes in public transit use (Feigon and Murphy, 2018), falling transit ridership following ride-hailing's entrance into a market (Malalgoda and Lim, 2019, Babar and Burtch, 2020, Graehler et al., 2019, Clewlow and Mishra, 2017), and that ride-hailing serves as a complement for the average transit agency (Hall et al., 2018). Notably, research also finds that transit may also substitute for ride-hailing trips, particularly during times of high ride-hailing demand (Grahn et al., 2020). To date, just one study has examined the association between ride-hailing and transit deserts: Jiao and Wang (2020) find less ride-hailing travel in transit deserts, which they suggest may be due to lower levels of ride-hailing vehicle supply or less awareness of ride-hailing services among groups that depend on transit.

Studies of ride-hailing travel across neighborhoods provide valuable insights into aggregate patterns of ride-hailing trips. Yet ride-hailing trip and aggregate data are typically divorced from individual traveler characteristics or trip-level details such as trip purpose. While a growing body of survey data research examines the nuances underlying 
aggregate ride-hailing patterns, findings remain murky about the potential for ride-hailing to provide increased levels of automobility to zero car and transit-dependent populations versus simply providing additional options for travelers who already enjoy high levels of mobility. Surveys of ride-hailing users rely varyingly on household travel diaries (Conway et al., 2018, Deka and Fei, 2019, Jiao and Wang, 2020), intercept surveys (Henao, 2017, Rayle et al., 2016), and online surveys of individual ride-hailing users (Masabi, 2018, Clewlow and Mishra, 2017, Dong, 2020). Surveys consistently find that ride-hailing users are more likely to be younger and car-less (Dong, 2020, Gehrke et al., 2019, Rayle et al., 2016, Conway et al., 2018). Survey research presents mixed results with respect to income, alternatingly finding that ride-hailing users earn disproportionately high (Conway et al., 2018, Clewlow and Mishra, 2017) and low incomes (Gehrke et al., 2019) compared to the general population. Dong (2020) finds that willingness to choose ride-hailing over transit is positively associated with income; each additional $\$ 1,000$ in annual household income corresponds to a $0.6 \%$ increased odds of a traveler choosing ride-hailing over transit. Less frequent transit riders are also more willing to substitute ride-hailing for transit than are frequent transit riders (Dong, 2020).

Survey data examining ride-hail-transit tradeoffs find that not only who is traveling matters, but so does the trip itself. Surveyed travelers, for example, report less willingness to take ride-hailing instead of transit for more expensive ride-hailing trips (Gehrke et al., 2019, Dong, 2020). Similar to aggregate data, survey data also report a higher share of ride-hailing travelers substituting ride-hailing for bus than they do for rail (Clewlow and Mishra, 2017). Some substitution may relate to quality of service; comparing identical trip pairs on transit versus ride-hailing in Chicago, Schweiterman \& Livingston (2018) find that 90 percent of trips could be made faster on non-shared ridehailing services than could be made on transit. While transit was more competitive when traveling to or from downtown, ride-hailing was far more competitive on average in part due to the high variation in walk times to transit (Schwieterman and Livingston, 2018). Similarly, Young et al. (2020) find that, in Toronto, transit trip duration often suffers from long walk and wait times. The authors find that nearly one-third (31\%) of ride-hailing trips in Toronto have viable transit alternative (less than 15 minutes travel time difference) while an additional 27 percent would have taken 30 or more minutes longer on transit. Young et al. (2020) conclude that the trip characteristics, rather than personal characteristics, most affect travel time savings on ride-hailing relative to transit, suggesting that the associations between ride-hailing and transit are unlikely to be uniform across time, place, or mode.

\subsection{Residential segregation and transit development in Chicago}

As we described earlier, the characteristics of the city are important to understand how transit and ride-hailing may interact. Chicago is the third largest city in the United States with a population of 2.7 million, and the economic center of a tri-state metropolitan area 
that has 9.5 million people. The majority of city residents are people of color; $7 \%$ of the population is Asian, $29 \%$ is Black or African American, $29 \%$ is Hispanic or Latino, and 33\% is white. Chicago is highly segregated by race and income, consistently ranked in the top five cities nationwide on indices of segregation (Acs et al., 2017). Residential segregation is not unique to Chicago, but scholars have argued that the foundations for de jure (and later de facto) segregation were laid here for at least two significant reasons. First, Chicago was an early leader in real estate innovations that promulgated segregation, such as racially restricted financing and housing covenants in the real estate market. These tools prevented Black people from buying homes outside a few select areas, and almost never in white neighborhoods. In cases where a Black family was able to purchase a home in a white neighborhood, real estate agents would often begin blockbusting, panicking neighbors that other people of color would soon move in and inciting an exodus of white homeowners (Lewinnek, 2014; Rothstein, 2017). Second, models of urban ecology describing racial and ethnic patterns of settlement in metropolitan areas were developed at the University of Chicago, and real estate developers took those models as the pseudoscientific basis to perpetuate racial discrimination in the housing market in the early part of the 20th century (Lewinnek, 2014).

Transit priorities have favored white and professional class residents in Chicago as well. For example, in the early 2000 s, city leadership proposed massive investment in rail infrastructure in the central area of the city, connecting commuter rail, linking the airports, and enhancing service around the central business district known as the Loop. These plans explicitly aimed to attract and retain workers in knowledge-based professions (Farmer, 2011). Meanwhile, a proposed extension of the Chicago Transit Authority (CTA) Red Line-which would bring additional rail service to the predominately Black south side of the city-has only recently been allocated money for pre-construction work after having languished for half a century. The effects of these conditions have meant that transit accessibility for marginalized groups falls well below that of whites. About half of all Black residents in the city live in the neighborhoods within the lowest tercile of transit access to jobs, grocery stores, and libraries. Neighborhoods with higher shares of Latino residents, low-income residents, and low educational attainment also have lower transit accessibility (Ermagun and Tilahun, 2020).

\section{Data and Methods}

Research remains mixed on whether ride-hailing serves as a complement or substitute to transit, whether ride-hailing fills mobility gaps in areas with high transit dependence but low transit supply, or whether there is a strong connection between ride-hailing and transit at all. Based on the review of the literature and the city context, we posit that levels of transit service in Chicago neighborhoods do influence the number of ride-hailing trips, but that the relationship will differ by neighborhood socioeconomic composition. In higher-income neighborhoods, we hypothesize that there would be more ride-hailing 
overall, regardless of transit service. These neighborhood residents can better afford ridehailing trips whether they use them to fill a travel need or in conjunction with transit. On the other hand, we expect to see more ride-hailing in poorer neighborhoods only when transit service is low; in other words, ride-hailing would be complementary to transit and fill a gap when cheaper alternatives are not available. Because of the patterns of segregation and development in the city, we expect to see stark geographic differences as well, with ride-hailing associated with racial and ethnic neighborhood composition.

The following sections detail the spatial descriptive statistics and multivariate analysis used to answer the research questions.

\subsection{Chicago context}

As we described earlier, people of color make up the majority of Chicagoans by race and ethnicity. The median household income is $\$ 57,238$ and $17.4 \%$ of the population lives below the poverty line. Transit is well-used: $28 \%$ of workers commuted by public transit in 2018. The Chicago Transit Authority (CTA) is the primary transit operator in the city, and operates an elevated rail system and a dense bus network. The agency served 468 million unlinked passenger trips in 2018,52\% of which were on buses (Federal Highway Administration, 2020). Other rail and bus agencies operating in Chicago (e.g. Metra and South Shore Line commuter rail services and Pace Bus) serve the suburbs and carry a small fraction of intracity passengers. Chicago was one of the first U.S. cities to have ridehailing services. Uber began its Chicago operations in 2011, while Lyft arrived in 2013 (Rao, 2011, Holly, 2017).

\subsection{Data and variables}

To understand the role that ride-hailing may play in filling transit service gaps, we gathered a combination of ride-hailing trip, transit, built environment, and job data. We used trip-level data from the City of Chicago Transportation Network Providers trip database (City of Chicago, 2020) to quantify ride-hailing travel. In November 2018, the city began requiring ride-hailing companies to routinely report trip-level data as a condition of their license to operate. Reported data include trip origin and destination by census tract or precise location, travel times, fares, and tips, among other variables. The city aggregates these data to census tracts and suppresses identifiers for locations outside city limits or when trip locations would compromise rider anonymity. Ride-hailing users in Chicago took over 128 million trips between November 1, 2018 and December 31,2019 . We used the full dataset to describe ride-hailing trip characteristics but used only the trips where both origin and destination census tracts are identified in spatial analyses ( $n=86,885,201$ trips).

We identified transit service gaps in census tracts using a measure of transit supply. A tract's overall transit supply score was a composite of four variables: transit stop 
density (number of stops per square kilometer), route density (route lengths in kilometers per square kilometer), median daily transit service headway, and the number of overnight (11 p.m.-5 a.m.) stops. We standardized each variable into a z-score and averaged all four scores to create the overall tract transit supply score. Measures of transit supply were obtained from the General Transit Feed Specification data for all transit agencies that operate in the City of Chicago, including the Chicago Transit Authority (CTA), Metra and South Shore Line (commuter rails), and Pace Bus (suburban bus operator). Not every agency provided all variables needed to construct the transit supply measure; in those instances, we used CTA data as the sole input. We used transit schedules in effect as of May 31, 2019-the midpoint of the study period-to calculate the transit supply measure. We used the composite transit score in descriptive and bivariate analyses but decomposed the score into its constituent parts for multivariate analyses. Separately, we identified census tracts that intersected with a $400 \mathrm{~m}$ buffer around rail stations to test effects of rail on ride-hailing. While larger buffers are often used when testing willingness to walk to rail stations, we chose a smaller buffer to understand ride-hailing trip generation and attraction at or near the rail stations themselves.

To characterize the neighborhoods in which both transit and ride-hailing operate, we obtained census tract-level demographic data from the 2014-2018 5-Year American Community Survey. Variables included basic sociodemographic characteristics, including percentage of population by race and ethnicity (white, Black, Asian, Latino), percentage of residents who are immigrants, and population density. We also included variables that measured transit dependence, including the percentage of households without a vehicle, percentage of young people (age 10-17), percentage of older people (over age 64), percentage unemployed, and median household income. We complemented demographic data with job density and job density by income category (low-, mid-, and high-wage) using data from the 2017 Longitudinal Employer-Household Dynamics (LEHD) survey. We used LEHD-defined job-income categories: low-wage jobs at \$1,250 per month or less, midwage jobs at $\$ 1,251-\$ 3,333$ per month, and high-wage jobs at more than $\$ 3,333$ per month. Summary statistics per census tract are shown in Table 1. 
TABLE 1 Summary statistics by census tract

\begin{tabular}{|c|c|}
\hline Characteristic & $\mathbf{N}=\mathbf{8 0 3 1}$ \\
\hline \multicolumn{2}{|l|}{ Ride-hailing trips } \\
\hline Pickups (all) & $133,463(400,864)$ \\
\hline Drop-offs (all) & $133,406(449,247)$ \\
\hline Pickups (weekday night) & $4,549(16,405)$ \\
\hline Drop-offs (weekday night) & $4,546(11,510)$ \\
\hline Pickups (weekend night) & $8,682(27,443)$ \\
\hline Drop-offs (weekend night) & $8,676(19,764)$ \\
\hline \multicolumn{2}{|l|}{ Socioeconomic characteristics } \\
\hline White population (\%) & $31(30)$ \\
\hline Black population (\%) & $36(40)$ \\
\hline Latino population (\%) & $26(29)$ \\
\hline Asian population (\%) & $6(9)$ \\
\hline Foreign-born population (\%) & $18(15)$ \\
\hline No household vehicles (\%) & $60(20)$ \\
\hline Population age $10-17$ (\%) & $9.1(4.5)$ \\
\hline Population age $65+(\%)$ & $12.3(6.4)$ \\
\hline Unemployed (\%) & $6.7(4.9)$ \\
\hline Median household income $(\$)$ & $57,295(32,221)$ \\
\hline Population density $\left(\mathrm{I} / \mathrm{km}^{2}\right)$ & $7,226(6,045)$ \\
\hline Low-wage employment density $\left(\mathrm{I} / \mathrm{km}^{2}\right)$ & $616(1,876)$ \\
\hline Mid-wage employment density $\left(\mathrm{I} / \mathrm{km}^{2}\right)$ & $882(2,823)$ \\
\hline High-wage employment density $\left(\mathrm{I} / \mathrm{km}^{2}\right)$ & $\mathrm{I}, 342(9,262)$ \\
\hline \multicolumn{2}{|l|}{ Transit characteristics } \\
\hline Transit stop density $\left(1 / \mathrm{km}^{2}\right)$ & $25(13)$ \\
\hline Transit route density $\left(\mathrm{I} / \mathrm{km}^{2}\right)$ & $8.1(7.4)$ \\
\hline Median transit headway (min) & $25(1 \mid 4)$ \\
\hline Number overnight stops & $175(185)$ \\
\hline Rail station within $400 \mathrm{~m}$ & $453(56 \%)$ \\
\hline
\end{tabular}

'Statistics presented: mean (SD); n (\%) 


\subsection{Methods}

\subsubsection{Descriptive analysis}

We examined ride-hailing pickups and drop-offs across the City of Chicago both overall and across five time periods that corresponded roughly to CTA transit schedules: (1) weekday daytime hours (5 a.m.-11 p.m.); (2) weekday overnight hours (11 p.m.-5 a.m.); (3) weekday peaks ( 6 a.m. -9 a.m., 4 p.m.-7 p.m.); (4) weekend daytime hours (5 a.m.-11 p.m.); and (5) weekend overnight hours (11 p.m.-5 a.m.). We identified hotspot and coldspot clusters of ride-hailing trip ends using the local Moran's I measure as an indicator of high or low spatial autocorrelation (Anselin, 1995). The local Moran's I statistic for observation $i$ is given by the following equation:

$$
I_{i}=c z_{i} \sum_{j} w_{i j} z_{j}
$$

where $z$ is the relevant variable's deviation from the mean, $w_{i j}$ is the spatial weight between census tracts $i$ and $j$, and $c$ is a scaling factor, ignorable for interpretation. Spatial weights were defined using queen contiguity for each neighbor; that is, census tracts that had a border in common in any direction were weighted as 1 , while non-neighbors were weighted as 0 . The algorithm for identifying statistically significant clusters distinguishes between values with large positive deviations from the mean surrounded by other high values (high-high or hotspots), values with large negative deviations from the mean surrounded by other low values (low-low or coldspots), and outliers of high values surrounded by low (high-low) and vice versa (low-high). A cluster is deemed significant when the observed I values are significantly different from I values generated by random permutations. We estimated the statistical significance of the local Moran's I using a false discovery rate correction for multiple comparisons, generally resulting in significance when $p<0.01$.

We also conducted a bivariate analysis to examine the spatial correlation of ridehailing trip ends compared to the sociodemographic variables and transit supply. We identified significant clusters and outliers using the bivariate version of local Moran's I ( $\left.I_{B}\right)$, whose interpretation is the same as the univariate version. The formula is similar,

$$
I_{B, i}=c x_{i} \sum_{j} w_{i j} y_{j}
$$

where $x$ and $y$ are standardized variables with a mean of zero and variance of one. We conducted separate analyses for ride-hailing pickups and drop-offs, though results were generally consistent for both trip ends. Given the temporal variation in transit supply, we compared the clustering of ride-hailing trips across the times of day described earlier.

\subsubsection{Multivariate analysis}

We next fit a series of multivariate spatial regression models to estimate the effects of transit availability on ride-hailing pickups and drop-offs per census tract. If ride-hailing 
were being used only to fill transit service gaps, we should see negative association between transit service and ride-hailing trip ends. If the two are complementary, the association should be positive. Likewise, if the transit-dependent population faced mobility limitations because of a lack of transit service, the association between those variables and ride-hailing trips should be positive if they used ride-hailing to make up for transit deficiency. Based on these hypotheses, we fit all models with the same independent variable specifications: control variables for race and foreign-born status, population density, variables for transit dependency detailed earlier, employment density by wage classification, and individual measures of transit supply including presence of a heavy rail station. While the composite $z$-score created earlier is a good overall measure of transit service for simpler analyses, the component variables indicate which, if any, specific characteristics of transit service are associated with ride-hailing trips. Model dependent variables were the logarithms of total ride-hailing pickups and drop-offs for three time periods: full day totals, weekday overnights, and weekend overnights. ${ }^{2}$

The regression models included all census tracts fully or partially within city limits except for three without population $(n=800)$ : the two international airports and a tract that contains mostly open space. We used the procedures suggested in Elhorst (2010) to determine the appropriate model structure, first estimating an aspatial ordinary least squares (OLS) regression with all trip ends as the dependent variable, then building up to different types of spatial models. We calculated variable inflation factors (VIF) to examine potential multicollinearity among the independent variables. None of the VIF values were greater than 8.4, and only logged household income and the percentages of Black residents, Latino residents, and immigrants had VIF values greater than 4 . We expected at least these variables to be significantly correlated because of the high degree of residential segregation in Chicago concentrated poverty in communities of color. Because they represent distinct demographic characteristics and because the coefficients were highly significant in the initial model specification, we retained all the variables.

The OLS model exhibited a significant degree of spatial autocorrelation (Moran's $I=0.21, p<0.001$ ). Lagrange Multiplier tests and null hypothesis tests rejected OLS in favor of spatial error and spatial lag models, and null hypothesis tests rejected both in favor of a spatial Durbin model (SDM). SDMs account for spatial dependence in two ways, via both endogenous and exogenous relationships with neighboring units (Yang et al., 2015, LeSage and Pace, 2009). For example, the number of ride-hailing pickups and dropoffs in a census tract is likely to be influenced by pickups and drop-offs in nearby tracts because ride-hailing drivers are routed to the nearest people requesting rides once they have completed their previous fare. And because of the patterns of residential segregation in Chicago, trip ends are likely to be influenced by demographic variables

\footnotetext{
${ }^{2}$ We also estimated models for peak and daytime hours, which performed similarly to the all-day models but are omitted here for brevity.
} 
both within the tract and by nearby tracts. The SDM in this study is given by the following equation:

$$
Y=\rho W Y+\alpha \iota_{N}+X \beta+W X \Theta+\varepsilon
$$

where $Y$ is the number of ride-hailing trip ends; $W$ is the spatial weights matrix indicating influence of neighbors; $X$ is the matrix of explanatory variables, including socioeconomic characteristics, employment type and density, and transit service levels with parameters $\beta$ to be estimated; $\iota_{N}$ is an $n \times 1$ vector of 1 s with intercept $\alpha$ to be estimated; $\rho$ is the spatial autoregressive term; and $\Theta$ is a vector of spatial effects of $W X$. The error term $\varepsilon$ is normally distributed with mean of 0 and variance of $\sigma^{2} I_{n}$ where $I_{n}$ is an identity matrix.

We initially fit the model with the logarithm of all trip ends as the dependent variable and all independent variables as described earlier using a queen-contiguous spatial weights matrix. Because the model still exhibited spatial autocorrelation after the first model fit, we tested multiple spatial weights matrices to eliminate it. The final model used a k-nearest neighbors weights matrix where $k=10$. We used the same model structure to estimate the six different quantities of interest.

In the main text of this paper, we present more interpretable effects from the regression models, partitioned into direct effects, that is, the contribution of census tract variables to the estimate, and indirect effects, or the influence on spatial neighbors. Clustering analyses were conducted in GeoDa (Anselin et al., 2010) while regression models were estimated using the spdep package (Bivand et al., 2013) in R (R Core Team, 2020). Full model coefficients are available in Appendix A.

\section{Results}

\subsection{Transit supply in Chicago}

Unsurprisingly, Chicago has better transit service (higher z-scores) in neighborhoods in and around the Loop and less robust supply farther away (Figure 1). Better scores follow the elevated CTA rail lines because of their generally frequent service, with lower service scores in areas between rail stops that are served only by bus. O'Hare International Airport, in the northwest of the city, has a low transit service score because although it has frequent service, the large land area covered by airport operations makes it impossible to have dense coverage in the census tract.

The correlation between transit access and sociodemographic characteristics is complex. At the citywide scale, we found limited evidence that transit supply was correlated with neighborhood characteristics such as race or income using non-spatial correlation analysis. Transit supply was instead more strongly correlated with employment density $(r=0.55)$. However, a spatial analysis reveals that the lack of correlation between sociodemographics and transit supply is due to substantial block-byblock differences rather than absence of inequity. For example, many census tracts in predominately Black areas on the west and south sides of Chicago are hotspots for high 
levels of transit service (Figure 2). But within those same broad geographic areas, clusters of low levels of transit service are interspersed between the clusters of high service. Similar patterns emerge when examining the Latino population and median household income. There were fewer outliers when examining the proportion of households without a vehicle, suggesting carless households are more likely to live near higher quality transit service.

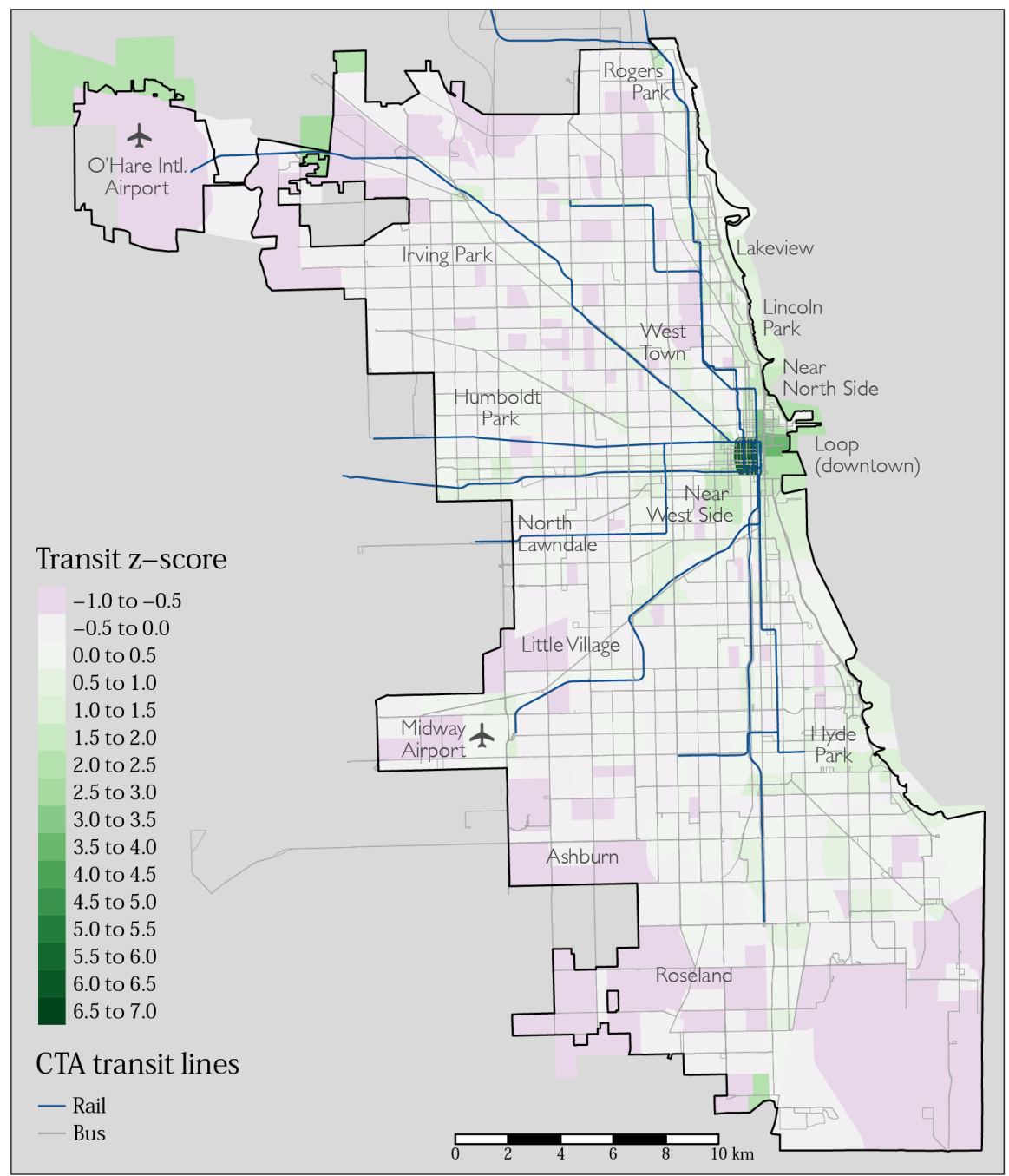

FIGURE 1 Distribution of transit service in Chicago (CTA = Chicago Transit Authority) 


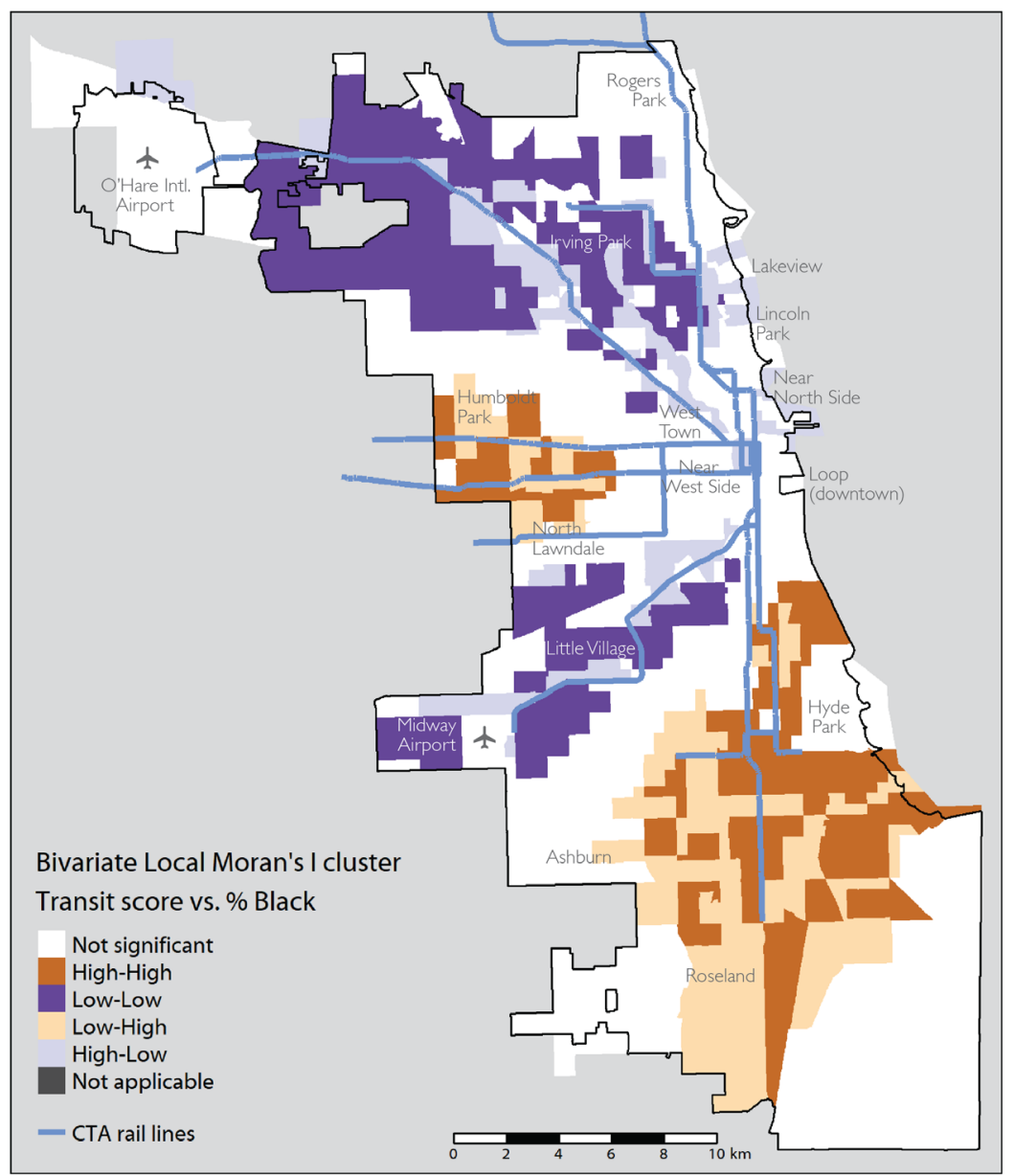

FIGURE 2 Bivariate transit service clusters with Black population

\subsection{Ride-hailing travel in Chicago}

Between November 1, 2018 and December 31, 2019, travelers completed more than 128 million ride-hailing trips in the city of Chicago, or nearly 289,000 trips on an average weekday and over 335,000 trips on an average weekend day (Table 2). The average trip distance was over six miles and cost about $\$ 12$, excluding tip. The share of trips during peak periods (28\%) and authorized as shared rides (21\%) is on-par with Lyft trips in Los Angeles (Brown, 2019a). Two-thirds of trips both started and ended within city limits.

The highest number of ride-hailing trips originated in the Near North Side community area; ${ }^{3} 21.2$ million trips (16.4\%) began in this entertainment district near downtown (Figure 3 ). The five other community areas with over $5 \%$ of trip origins included the Loop, Chicago's central business district (9.0\%); the Near West Side, which includes residential, commercial, and university uses (8.7\%); Lake View, a primarily

\footnotetext{
${ }^{3}$ Community areas are official designations that often-but do not always-correspond with neighborhoods as thought of by residents. We use the community area geographies in this section and the names descriptively elsewhere.
} 
residential neighborhood along Lake Michigan (6.6\%); and the gentrifying community areas of West Town (6.2\%) and Lincoln Park (5.4\%). O'Hare International Airport had $3.6 \%$ of trip origins. Destination patterns were similar. The Near North Side had 20.1 million trips end within the community area (15.6\%), followed by the Loop (9.9\%), the Near West Side (8.8\%), Lake View (6.5\%), West Town (5.7\%), Lincoln Park (5.2\%), and O'Hare (4.4\%). The remainder of the community areas accounted for fewer than $4 \%$ of trip destinations each.
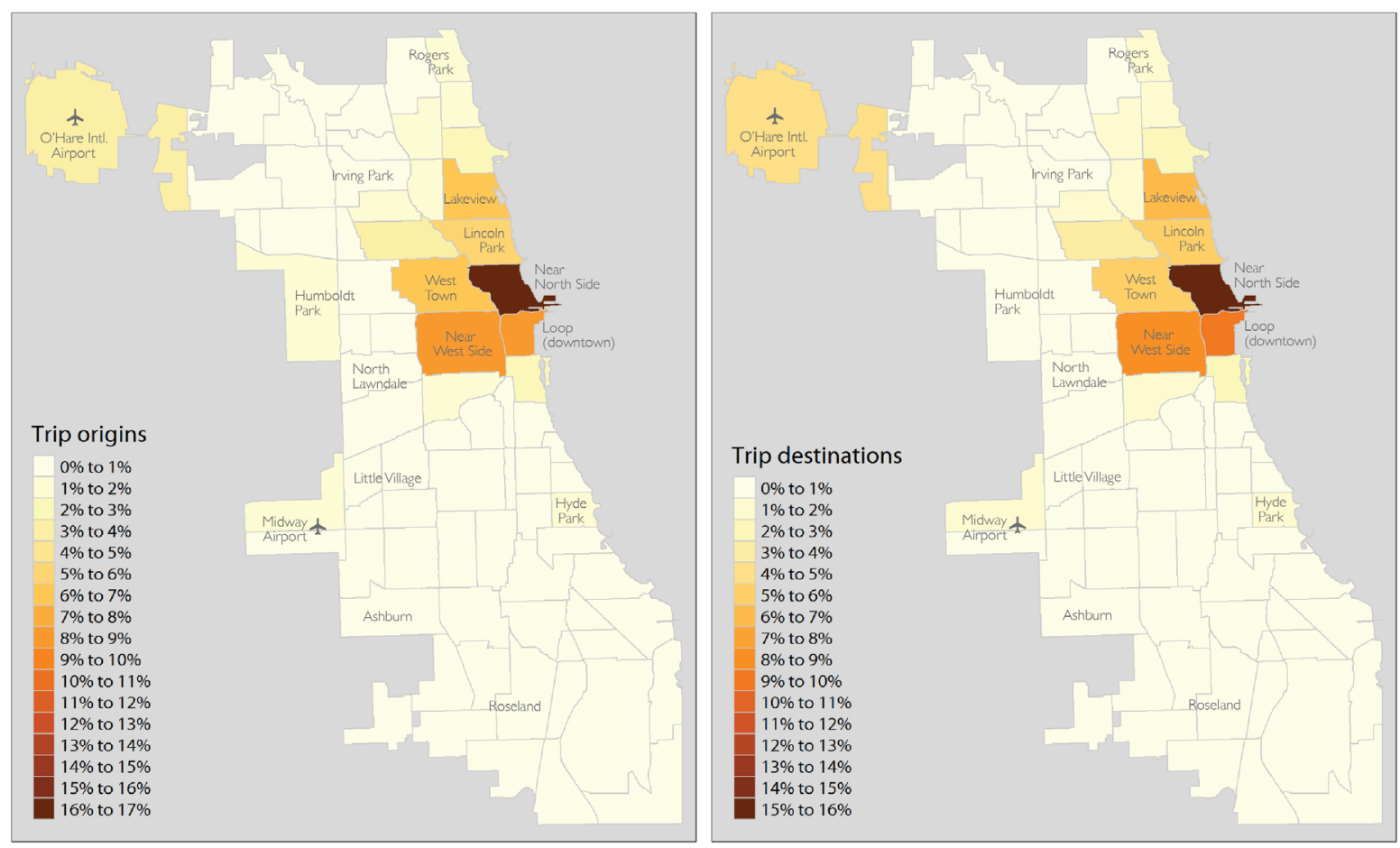

FIGURE 3 Ride-hailing Trip Origins and Destinations 
TABLE 2 Chicago Ride-hailing Statistics, November 1, 2018 to December 31, 2019

\begin{tabular}{lr}
\hline Variable & Mean (SD) or \% \\
\hline Trip distance $(\mathrm{mi})$ & $6.2 \mathrm{I}(6.94)$ \\
Trip time $(\mathrm{min})$ & $\mathrm{I} 8.2(13 . \mathrm{I})$ \\
Base fare $(\$)$ & $\mathrm{I} .96(10.46)$ \\
Total fare (\$) & $15.58(12.4 \mathrm{I})$ \\
Trips in peak period' & $28 \%$ \\
Shared trip authorized & $21 \%$ \\
Trips per weekday & $288,882(48,90 \mathrm{I})$ \\
Trips per weekend day & $335,250(54,807)$ \\
Share of trips within Chicago & $67 \%$ \\
N & $128,720,757$ \\
\hline Note I: Peak = weekdays, 6 a.m.-9 a.m., \\
4 p.m. - 7 p.m.
\end{tabular}

Source: City of Chicago (2020)

A significant fraction of ride-hailing trip flows within Chicago takes place in the urban core. The most common origin-destination pair is trips within the Near North Side (4.7\%), followed by trips between the Near North Side and the Loop (2.9\% to the Loop, $2.4 \%$ from the Loop). High trip flows also exist between these two neighborhoods and the adjacent Near West Side. Trips between these three neighborhoods-which represent just $4 \%$ of Chicago's 77 community areas and $7 \%$ of the population, and include the central business district and adjacent neighborhoods-accounted for over one out of every six ride-hailing trips within the city during the 14-month study period.

The trip flow patterns suggested a significant concentration of trips within neighborhoods, which we identified when examining trip end clusters by census tract (that is, both pickups and drop-offs; see Figure 4). High clusters of trip drop-offs are concentrated in central Chicago, including the Loop, the Near North Side, and neighborhoods west of downtown, which include transportation hubs and the University of Illinois at Chicago. There are several non-contiguous clusters of a low number of trip ends along the west and south sides of Chicago. These include low-income and predominately Black and Latino neighborhoods in addition to light-industrial and manufacturing areas. Comparatively fewer higher-income and majority white neighborhoods on the north and northwest sides of the city have low clusters of trip ends. A single outlier, with a high number of trip ends relative to surrounding areas, contains Midway International Airport, located in the otherwise residential and local retail-heavy Garfield Ridge neighborhood. For trip drop-offs, an additional outlier cluster is located north of O'Hare International Airport. (Only a portion of this census tract falls within the city limits.) 

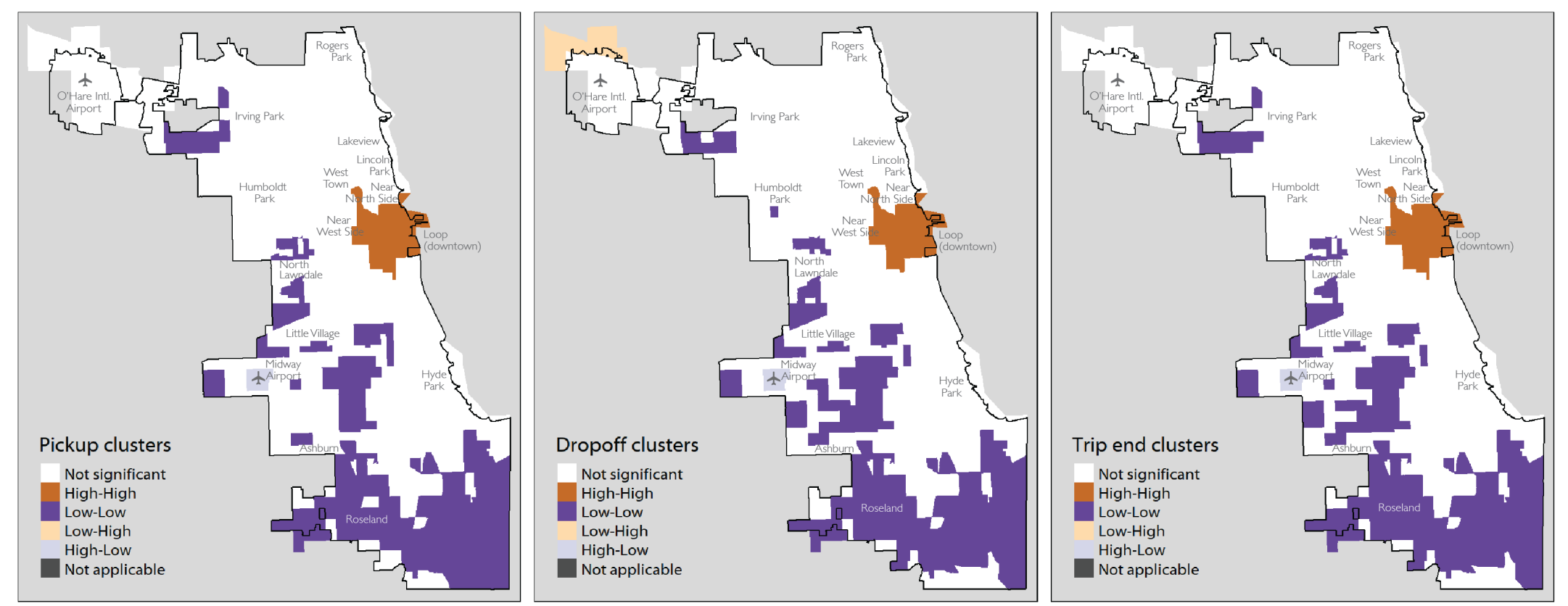

FIGURE 4 Ride-hailing pickup (left), dropoff (center) and trip end (right) clusters. Dark orange indicates a cluster with a high number of trip ends, dark purple indicates a cluster with a low number of trip ends. Outliers are at Midway International Airport (light purple) and O'Hare International Airport (light orange). 
Areas with high and low trip-making clusters vary across times of day and days of the week. For example, on weekday nights (11 p.m. - 5 a.m.), additional clusters of high trip drop-offs were present in farther north side neighborhoods and areas west of the Loop. The coldspot clusters shown in Figure 4 expanded in west and south side neighborhoods during the same overnight period (not shown). While trip clustering during weekend days virtually mirrored weekday days, trip clusters were significantly expanded during weekend nights. High trip end areas expanded further into north side residential neighborhoods while low trip end clusters included almost the entire south side of Chicago and several more west side neighborhoods.

Clusters of high and low ride-hailing trip ends were correlated with neighborhood racial and ethnic characteristics (Figure 5). The maps show several clustering patterns: light purples and dark oranges indicate high clustering by race or ethnicity, while dark purples and light oranges indicate low clustering. Purples indicate low numbers of ridehailing trip ends and oranges indicate high numbers. The maps highlight, first, the stark segregation of racial and ethnic groups in Chicago. Very few areas exist where a high proportion of Black residents corresponds to a high proportion of ride-hailing trip ends except for the census tracts including and surrounding the Cabrini Green public housing development. Similarly, only one census tract has a significantly high share of Latino residents and a high number of trip ends. Notably, there were no census tracts in the lowhigh category for the white population; in other words, nowhere did clusters of a low percentage of white residents correspond with a high number of ride-hailing pickups and drop-offs.

This is the author's accepted manuscript of an article published in the Journal of Transport Geography, available at https://doi.org/10.1016/j.jtrangeo.2020.102918. This manuscript version is made available under the CC-BY-NC-ND 4.0 license http://creativecommons.org/licenses/by-nc-nd/4.0/ 

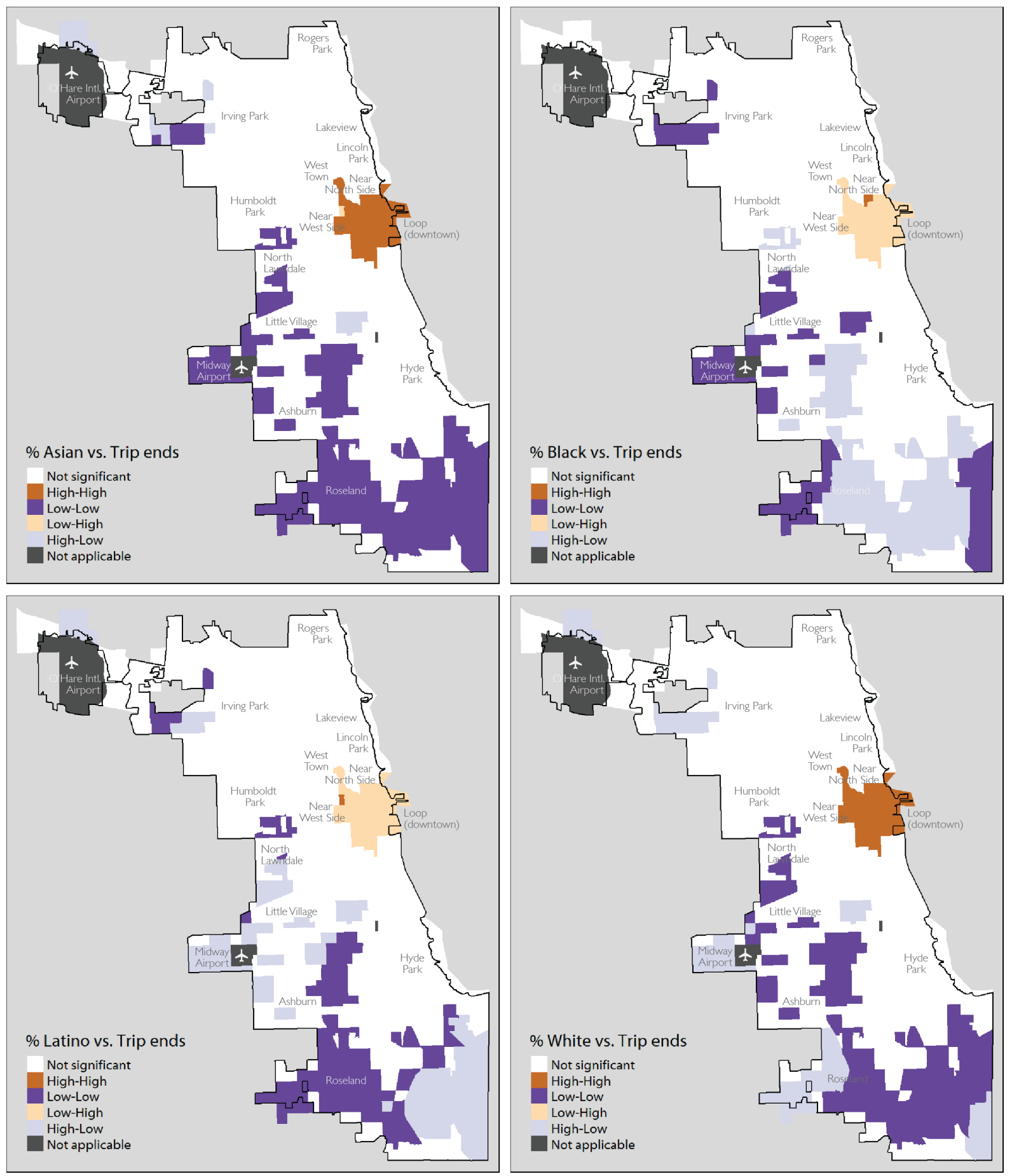

FIGURE 5 Bivariate clusters of ride-hailing trip ends with percent race/ethnicity. Tracts home to O'Hare and Midway Airports display as "not applicable" as they are home to zero population. 


\subsection{Does Ride-hailing fill transit gaps?}

We found little evidence in bivariate analyses that ride-hailing trips are associated with a low level of overall transit service. In other words, ride-hailing trips are not significantly substituting for travel in transit deserts. There were few statistically significant clusters of ride-hailing trip ends with the transit service score defined earlier (Figure 6). As before, we found high-high clustering in central Chicago census tracts, meaning that areas with the most transit service also had the highest number of ride-hailing pickups and drop-offs. The census tracts near O'Hare International Airport in the northwest of the city are coldspots for both transit service and ride-hailing trips, as are neighborhoods in the extreme southwest and southeast portions of the city. Only three census tracts were defined in a cluster of high ride-hailing trip ends and low transit service, located in a mixed residential-commercial neighborhood northwest of the Loop. No neighborhoods were in clusters of low ride-hailing trips but high transit service. The clustering patterns did not meaningfully change when examining ride-hailing trips categorized by time of day. 


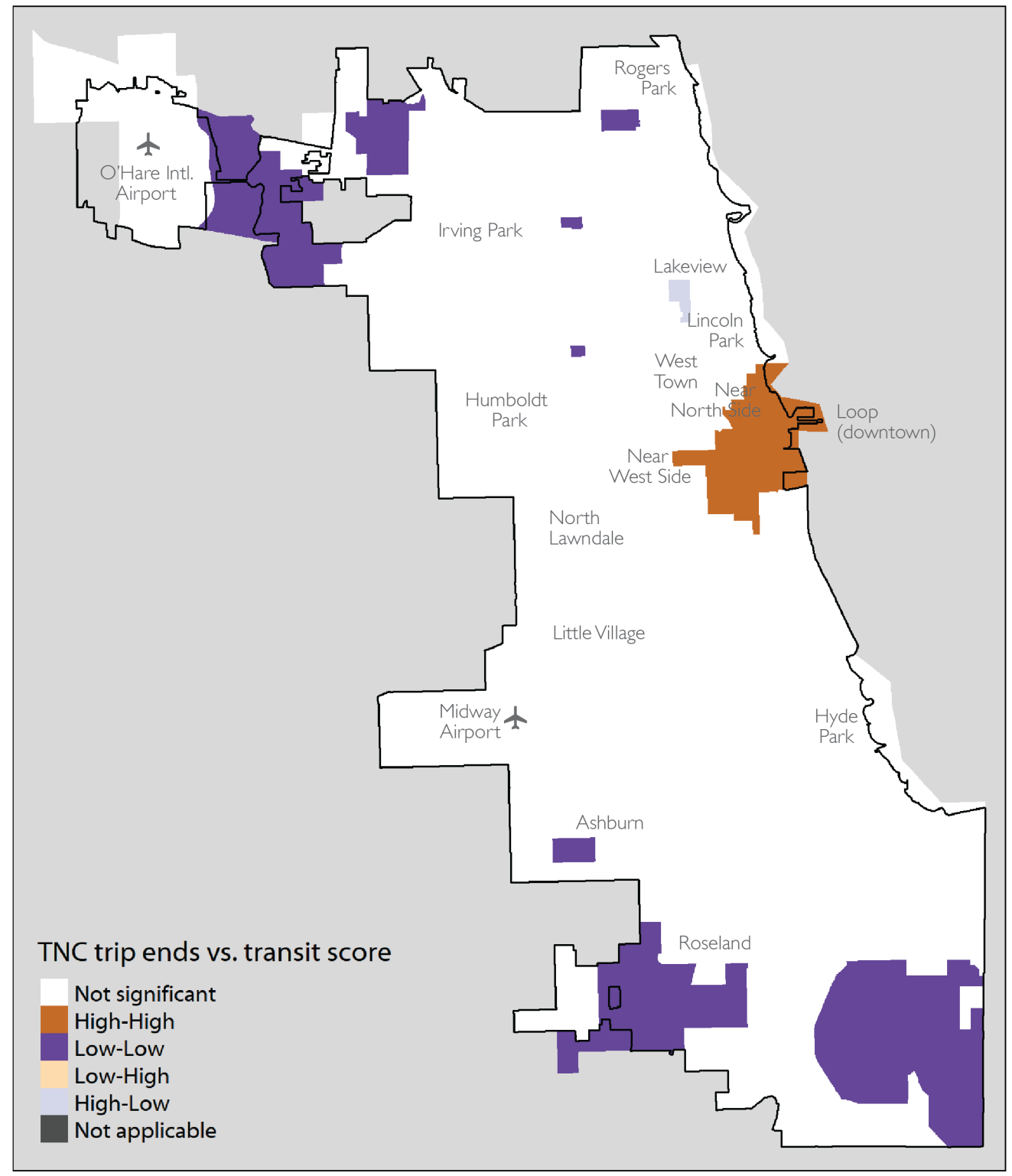

FIGURE 6 Bivariate clusters of ride-hailing trip ends with transit service score.

The relationship between transit service and ride-hailing trips changed somewhat when examining individual service characteristics. Model results showed that the overall total effects of transit supply on ride-hailing pickups and drop-offs were insignificant, meaning that transit service did not have a relationship with ride-hailing trips when including the cluster of nearby neighboring census tracts. However, some of the direct effects - that is, characteristics within the census tract itself-were significant. Table 3 shows the decomposed effects from the multivariate models. (Full model coefficients are available in Appendix A, Table A.1.) In general, the effects of all model independent variables on ride-hailing trips were similar in character and magnitude for both ridehailing pickups and drop-offs. Neither route density nor transit headways were 
significantly associated with the number of pickups or drop-offs within a tract. However, higher transit stop density, whose value is dominated by the presence of bus stops, was associated with a lower number of ride-hailing trip ends. For every $10 \%$ increase in the number of transit stops per unit area, ride-hailing pickups decreased by $1.9 \%$ and ridehailing drop-offs decreased by $2.1 \%$. Overnight transit service was significantly associated with ride-hailing trips: for every 10 additional stops in a tract, the number of ride-hailing pickups and drop-offs increased by $2 \%$. Ride-hailing trip ends in nearby tracts decreased with a greater number of overnight stops, suggesting concentrations of overnight activity exists within clusters. The largest effect on ride-hailing trips was whether a rail station was located in or nearby a census tract. Tracts within walking distance of rail stations (400 m) had $18 \%$ more pickups and $22 \%$ more drop-offs than those not near rail.

The greatest socioeconomic effect on ride-hailing trips per neighborhood was median household income. A $10 \%$ increase in median income in a tract was associated with a $4.1 \%$ increase in ride-hailing pickups and a $3.7 \%$ increase in drop-offs. The indirect effect on neighboring tracts was over five times that. In other words, a high-income neighborhood saw substantially more pickups clustered, all else equal. Other socioeconomic variables were less influential but many were significant. A higher proportion of households without vehicles was associated with more ride-hailing pickups. A slightly positive total association existed between the proportion of people of color and ride-hailing pickups. Much of this relationship was significant for indirect effects; more people of color in a neighborhood predicted more ride-hailing trips for neighboring tracts. Tracts with a higher percentage of carless households had more ride-hailing trips, while tracts with higher unemployment rates had fewer trips. Areas with higher shares of both teenagers and seniors had fewer ride-hailing trips. Only adults aged 18 or older are permitted to sign up for ride-hailing services, limiting utility at the lower end of the age spectrum, while older adults may be less likely to use services because of cost, safety, privacy, and lack of familiarity with technology-based services (Shirgaokar, 2020).

Employment had some association with ride-hailing trips. Low-wage employment density was a significant predictor of both pickups and drop-offs, but high-wage employment density was significant only for drop-offs and to a much smaller degree. Lowwage employment was a better predictor of clusters of nearby ride-hailing trips. Total employment density was also significantly associated with ride-hailing trips in additional models tested (but not shown here). A $10 \%$ increase in jobs per unit area yielded a $0.3 \%$ increase in trips ends per tract.

The models revealed a degree of variation in associations between ride-hailing and transit supply across different time periods, though most associations were consistent with the models estimating ride-hailing trip totals (Appendix A, Table A.2). Transit stop density was associated with fewer overnight ride-hailing pickups and drop-offs, but had significant indirect positive effects on weekend night pickups in neighboring tracts. The number of overnight transit stops was positively associated with both ride-hailing origins and destinations, while presence of a rail station became marginally significant for 
weekday overnight pickups and weekend overnight drop-offs, and insignificant for weekday night drop-offs. 


\begin{tabular}{|c|c|c|c|c|c|c|}
\hline & \multicolumn{3}{|c|}{ Pickups (all) } & \multicolumn{3}{|c|}{ Drop-offs (all) } \\
\hline & Direct & Indirect & Total & Direct & Indirect & Total \\
\hline Black population (\%) & -0.001 & 0.034 & 0.033 & -0.002 & 0.033 & 0.031 \\
\hline Latino population (\%) & -0.007 & 0.023 & 0.016 & -0.010 & 0.024 & 0.014 \\
\hline Asian population (\%) & 0.003 & 0.044 & 0.047 & -0.001 & 0.048 & 0.047 \\
\hline Foreign-born population (\%) & -0.004 & 0.016 & 0.012 & -0.002 & 0.012 & 0.010 \\
\hline No household vehicles (\%) & 0.015 & 0.011 & 0.025 & 0.015 & 0.009 & 0.024 \\
\hline Population age 10-17 (\%) & -0.014 & -0.133 & -0.147 & -0.017 & -0.126 & -0.143 \\
\hline Population age $65+(\%)$ & -0.010 & $-0.08 I$ & -0.090 & -0.011 & -0.077 & -0.088 \\
\hline Unemployed (\%) & -0.031 & 0.015 & -0.015 & -0.032 & 0.012 & -0.020 \\
\hline Median household income (log \$) & $0.4 I I$ & 1.382 & 1.793 & 0.366 & 1.295 & 1.661 \\
\hline Population density (log) & 0.122 & 0.028 & 0.150 & -0.003 & 0.113 & 0.110 \\
\hline Low-wage employment density (log) & 0.177 & 0.352 & 0.529 & 0.189 & 0.378 & 0.567 \\
\hline High-wage employment density (log) & 0.050 & 0.007 & 0.057 & 0.064 & 0.002 & 0.066 \\
\hline Transit stop density (log) & -0.188 & 0.332 & 0.144 & -0.214 & 0.385 & 0.171 \\
\hline Transit route density (log) & -0.057 & 0.383 & 0.326 & 0.022 & 0.197 & 0.219 \\
\hline Median transit headway (min) & 0.000 & 0.000 & 0.000 & 0.000 & 0.000 & 0.000 \\
\hline Number overnight stops & 0.002 & -0.002 & 0.000 & 0.002 & -0.002 & 0.000 \\
\hline Rail station within $400 \mathrm{~m}$ & 0.182 & -0.248 & -0.066 & 0.223 & -0.196 & 0.027 \\
\hline
\end{tabular}

Note: Bold indicates $p<0.05$, italics $p<0.10$

\section{TABLE 3 Ride-hailing Models Effects}




\section{Discussion}

The geographies of both transit service and ride-hailing trips are uneven in urban areas, but the unevenness of the two modes can be complementary if ride-hailing fulfills mobility needs that transit cannot. Using publicly available datasets of ride-hailing trips and transit service in Chicago, we found that ride-hailing is not likely being used to fill gaps in transit service. A relative lack of transit service is spatially correlated with some indicators of socioeconomic disadvantage, including race and ethnicity, household income, and car availability. But even within the low service areas, there are pockets of high transit availability and vice versa. Ride-hailing trips were more strongly clustered with markers of advantage, and the median household income of a census tract was the strongest socioeconomic predictor of the number of ride-hailing pickups and drop-offs. In bivariate analyses, transit service was not associated with ride-hailing trip ends. But after controlling for neighborhood socioeconomic status, transit dependency, population density, and employment density, we found that certain transit characteristics were associated. In neighborhoods were bus service dominated, there were fewer ride-hailing trips, but where rail service was prevalent, there was significantly more ride-hailing. Patterns were slightly different for overnight weekend ride-hailing pick-ups, where higher transit density predicted a greater number of trips in nearby tracts. These findings are similar to those of Jiao and Wang (2020), who found that in New York City, shared mobility services concentrate in areas with better transit and a smaller transit-dependent population.

The ride-hailing and transit patterns revealed in this analysis could be a result of several factors. First, people who live in lower-income neighborhoods or who are dependent on transit may not be aware of ride-hailing services (Jiao and Wang, 2020), or may not be able to afford to use ride-hailing regularly regardless of whether transit service is adequate for their needs. This possibility is reflected in surveys of ride-hailing users, who some researchers find are higher-income compared to the general population (Conway et al., 2018). Even if neighborhood transit service is poor, lower-income people may just choose to not to make a trip rather than use a ride-hailing alternative. And while the relationship between higher low-wage employment density and more ride-hailing trips may seem to indicate that lower-income individuals may use ride-hailing for certain trips more, it is more likely to indicate that places dominated by service and retail activities are attracting trips (Brown, 2019a). Although the models are based on aggregate data and therefore run afoul of the ecological fallacy when suggesting neighborhood-level results are true for individual travel behavior, Chicago remains one of the most highly segregated U.S. cities (Acs et al., 2017) and it is more likely that individuals in a tract match the aggregate.

Second, trip patterns around neighborhoods of color could reflect discrimination against Black, Latino, and Asian riders. Although the models did not indicate a relationship between ride-hailing and race or ethnicity within a census tract, they did suggest that a 
higher share of people of color predicts more ride-hailing in neighboring tracts. In other words, ride-hailing trips are more likely to take place in zones where racial composition is mixed, rather than in highly segregated communities of color. There is some evidence that ride-hailing drivers cancel requests more frequently for riders with stereotypically Blacksounding names (Ge et al., 2016), although other researchers actually find more ridehailing trips in majority-black neighborhoods compared to neighborhoods with no racial or ethnic majority (Brown, 2019a). Note, however, that our findings suggest income rather than race or ethnicity is a better predictor of ride-hailing.

Third, it is possible that ride-hailing trips and transit are indeed complementary services in neighborhoods of high activity. This is supported by the positive associations between both rail and overnight transit service and ride-hailing. Especially at night, ridehailing provides a door-to-door option that users-particularly women-might consider to be safer compared to transit (Marshall, 2018), while overnight transit provides an affordable lifeline for those who need to travel. Unfortunately, we cannot know from the data whether ride-hailing riders are taking trips as first-mile or last-mile connections to transit or if they are replacing what would have been a transit trip, only that there is a high demand for transportation of any mode.

Transit and ride-hailing service also compete with one another, and so planners and policymakers must carefully consider how the two modes should work together as part of a solution to close transit service gaps. From an environmental and economic equity perspective, investment in high-quality, frequent, reliable transit is preferable to a solution that encourages predominately single-rider trips in areas of low service but relatively high need. But universal transit coverage is a difficult goal to obtain, and finding ways to encourage ride-hailing drivers to serve underserved areas could serve broader transportation objectives. For example, cities could allow ride-hailing companies to go beyond a cap on permits, or reduce trip taxes or fees, if they meet or exceed established metrics for the total share or number of trips in areas of low transit supply. Cities have already pursued similar strategies for modes such as shared e-scooters (e.g. Portland Bureau of Transportation, 2019). A complementary policy to subsidize rides for lowincome users with some restrictions-for example, only in times when transit is not running or to connect to transit hubs-could also help ensure that the two modes remain mutually beneficial.

\section{Conclusion}

This research sought to understand if ride-hailing fills transit service needs gaps. To do so, we examined the spatial distribution of public transit service and ride-hailing trips with respect to neighborhood racial, ethnic, socioeconomic, and built environment characteristics, and with respect to each other. We found small to insignificant correlations between transit service and most neighborhood characteristics, but significant evidence that ride-hailing trips ends are clustered in predominately white 
neighborhoods. Ride-hailing trips are not associated with a lack of transit service, but are instead associated with higher levels of transit stop density and overnight service, as well as with median household income.

Additional monitoring and research are needed to uncover the root causes behind lower levels of ride-hailing use in low-income neighborhoods. Results are not necessarily evidence of discrimination or exclusion: fewer trips could result from either lack of demand or lack of supply (or both). For example, existing modes-transit, walking, biking, and personal cars-may meet residents' current travel needs. But another explanation is that a combination of discrimination (see for example Brown (2019b)) or financial or technological barriers prevent access to ride-hailing services. Other research has found that lower-income travelers have substantially lower access to the "smart mobility ecosystem," including bank accounts, and remain disproportionately cash dependent, and face mobile data limitations (Golub et al., 2019). Additional research is needed to inform planning and policy that ensures that ride-hailing services expand travel options to those who need them the most.

\section{Acknowledgements}

This research did not receive any specific grant from funding agencies in the public, commercial, or not-for-profit sectors.

\section{References}

ACS, G., PENDALL, R., TRESKON, M. \& KHARE, A. 2017. The cost of segregation: National Trends and the Case of Chicago, 1990-2010. Urban Institute. March. Urban Institute.

AL MAMUN, M. S. \& LOWNES, N. E. 2011a. Measuring service gaps: accessibility-based transit need index. Transportation Research Record, 2217, 153-161.

AL MAMUN, M. \& LOWNES, N. E. 2011b. A composite index of public transit accessibility. Journal of Public Transportation, 14, 4.

ANSELIN, L. 1995. Local indicators of spatial association-LISA. Geographical analysis, 27, 93-115.

ANSELIN, L., SYABRI, I. \& KHO, Y. 2010. GeoDa: an introduction to spatial data analysis. Handbook of applied spatial analysis. Springer.

BABAR, Y. \& BURTCH, G. 2020. Examining the Heterogeneous Impact of Ride-Hailing Services on Public Transit Use. Information Systems Research.

BIVAND, R., PEBESMA, E. \& GOMEZ-RUBIO, V. 2013. Applied spatial data analysis with $R$, New York, Springer.

BROWN, A. 2019a. Redefining Car Access: Ride-Hail Travel and Use in Los Angeles. Journal of the American Planning Association, 85, 83-95. 
BROWN, A. E. 2019b. Prevalence and Mechanisms of Discrimination: Evidence from the Ride-Hail and Taxi Industries. Journal of Planning Education and Research, $0739456 \times 19871687$.

CARLETON, P. R. \& PORTER, J. D. 2018. A comparative analysis of the challenges in measuring transit equity: definitions, interpretations, and limitations. Journal of transport geography, 72, 64-75.

CITY OF CHICAGO. 2020. Transportation Network Providers - Trips [Online]. Available: https://data.cityofchicago.org/Transportation/Transportation-NetworkProviders-Trips/m6dm-c72p [Accessed July 1 2019].

CLEWLOW, R. R. \& MISHRA, G. S. 2017. Disruptive Transportation: The Adoption, Utilization, and Impacts of Ride-Hailing in the United States. UC Davis Institute of Transportation Studies.

CONWAY, M., SALON, D. \& KING, D. 2018. Trends in Taxi Use and the Advent of Ridehailing, 1995-2017: Evidence from the US National Household Travel Survey. Urban Science, 2, 79.

CURRIE, G. 2004. Gap analysis of public transport needs: measuring spatial distribution of public transport needs and identifying gaps in the quality of public transport provision. Transportation Research Record, 1895, 137-146.

CURRIE, G. 2010. Quantifying spatial gaps in public transport supply based on social needs. Journal of Transport Geography, 18, 31-41.

DEKA, D. \& FEI, D. 2019. A comparison of the personal and neighborhood characteristics associated with ridesourcing, transit use, and driving with NHTS data. Journal of Transport Geography, 76, 24-33.

DELBOSC, A. \& CURRIE, G. 2011. Using Lorenz curves to assess public transport equity. Journal of Transport Geography, 19, 1252-1259.

DONG, X. 2020. Trade Uber for the Bus? An Investigation of Individual Willingness to Use Ride-Hail Versus Transit. Journal of the American Planning Association, 86, 222-235.

ERMAGUN, A. \& TILAHUN, N. 2020. Equity of transit accessibility across Chicago. Transportation Research Part D: Transport and Environment, 86, 102461.

EL-GENEIDY, A., GRIMSRUD, M., WASFI, R., TÉTREAULT, P. \& SURPRENANT-LEGAULT, J. 2014. New evidence on walking distances to transit stops: identifying redundancies and gaps using variable service areas. Transportation, 41, 193-210.

ELHORST, J. P. 2010. Applied spatial econometrics: raising the bar. Spatial economic analysis, 5, 9-28.

FARMER, S. 2011. Uneven Public Transportation Development in Neoliberalizing Chicago, USA. Environ Plan A, 43, 1154-1172.

FAYYAZ, S. K., LIU, X. C. \& PORTER, R. J. 2017. Dynamic transit accessibility and transit gap causality analysis. Journal of Transport Geography, 59, 27-39.

FEDERAL HIGHWAY ADMINISTRATION 2020. The National Transit Database.

FEIGON, S. \& MURPHY, C. 2018. Broadening Understanding of the Interplay Between Public Transit, Shared Mobility, and Personal Automobiles. 
Transportation Cooperative Research Program. Washington, D.C.: Transportation Research Board.

FRANSEN, K., NEUTENS, T., FARBER, S., DE MAEYER, P., DERUYTER, G. \& WITLOX, F. 2015. Identifying public transport gaps using time-dependent accessibility levels. Journal of Transport Geography, 48, 176-187.

GE, Y., KNITTEL, C. R., MACKENZIE, D. \& ZOEPF, S. 2016. Racial and Gender Discrimination in Transportation Network Companies. National Bureau of Economic Research.

GEHRKE, S. R. 2020. Uber service area expansion in three major American cities. Journal of Transport Geography, 86, 102752.

GEHRKE, S. R., FELIX, A. \& REARDON, T. G. 2019. Substitution of ride-hailing services for more sustainable travel options in the greater Boston region. Transportation Research Record, 2673, 438-446.

GLAESER, E. L., KAHN, M. E. \& RAPPAPORT, J. 2008. Why do the poor live in cities? The role of public transportation. Journal of Urban Economics, 63, 1-24.

GOLUB, A., SATTERFIELD, V., SERRITELLA, M., SINGH, J. \& PHILLIPS, S. 2019. Assessing the barriers to equity in smart mobility systems: A case study of Portland, Oregon. Case Studies on Transport Policy, 7, 689-697.

GRAEHLER, M., MUCCI, R. A. \& ERHARDT, G. D. Understanding the recent transit ridership decline in major US cities: service cuts or emerging modes. 98th Annual Meeting of the Transportation Research Board (TRB), Washington DC, 2019.

GRAHN, R., QIAN, S., MATTHEWS, H. S. \& HENDRICKSON, C. 2020. Are travelers substituting between transportation network companies (TNC) and public buses? A case study in Pittsburgh. Transportation, 1-29.

HALL, J. D., PALSSON, C. \& PRICE, J. 2018. Is Uber a substitute or complement for public transit? Journal of Urban Economics, 108, 36-50.

HENAO, A. 2017. Impacts of Ridesource - Lyft and Uber - on Transportation including VMT, Mode Replacement, Parking, and Travel Behavior. Doctoral Dissertation. Denver: University of Colorado, Denver.

HOLLY, R. 2017. Lyft just opened a new Chicago office - take a look inside. Chicago Tribune.

JIAO, J. 2017. Identifying transit deserts in major Texas cities where the supplies missed the demands. Journal of Transport and Land Use, 10, 529-540.

JIAO, J. \& DILLIVAN, M. 2013. Transit deserts: The gap between demand and supply. Journal of Public Transportation, 16, 2.

JIAO, J. \& WANG, F. 2020. Shared mobility and transit-dependent population: A new equity opportunity or issue? International Journal of Sustainable Transportation, 1-12.

LAVIERI, P. S., DIAS, F. F., JURI, N. R., KUHR, J. \& BHAT, C. R. 2018. A model of ridesourcing demand generation and distribution. Transportation Research Record, 0361198118756628. 
LESAGE, J. \& PACE, R. K. 2009. Introduction to spatial econometrics, Chapman and Hall/CRC.

LEWINNEK, E. 2014. The working man's reward: Chicago's early suburbs and the roots of American sprawl, Oxford Univ. Press, Oxford.

MALALGODA, N. \& LIM, S. H. 2019. Do transportation network companies reduce public transit use in the US? Transportation Research Part A: Policy and Practice, 130, 351372.

MANVILLE, M., TAYLOR, B. D. \& BLUMENBERG, E. 2018. Falling Transit Ridership: California and Southern California. UCLA Institute of Transportation Studies.

MARSHALL, A. 2018. The Pink Transit Tax: Women Spend More than Men to Get Around NYC. Wired.

MASABI 2018. Mass Transit Rider Research Report: Key Factors Influencing Ridership in North America.

MAVOA, S., WITTEN, K., MCCREANOR, T. \& O'SULLIVAN, D. 2012. GIS based destination accessibility via public transit and walking in Auckland, New Zealand. Journal of transport geography, 20, 15-22.

PORTLAND BUREAU OF TRANSPORTATION 2019. TRN-15.01 - New Mobility - Shared Electric Scooters. In: TRANSPORTATION, P. B. O. (ed.).

$R$ CORE TEAM 2020. $R$ : A language and environment for statistical computing. $R$ Foundation for Statistical Computing, Vienna, Austria.

RAO, L. 2011. Uber Brings Its Disruptive Car Service To Chicago. Tech Crunch.

RAYLE, L., DAI, D., CHAN, N., CERVERO, R. \& SHAHEEN, S. 2016. Just a better taxi? A survey-based comparison of taxis, transit, and ridesourcing services in San Francisco. Transport Policy, 45, 168-178.

ROTHSTEIN, R. 2017. The color of law: a forgotten history of how our government segregated America, Liveright Publishing Corporation, New York ; London.

SABOURI, S., PARK, K., SMITH, A., TIAN, G. \& EWING, R. 2020. Exploring the influence of built environment on Uber demand. Transportation Research Part D: Transport and Environment, 81, 102296.

SANCHEZ, T.W. 1999. The connection between public transit and employment. Portland. Portland: Portland State University Center for Urban Studies.

SCHWIETERMAN, J. P. \& LIVINGSTON, M. 2018. Uber Economics: Evaluating the Monetary and Nonmonetary Tradeoffs of TNC and Transit Services in Chicago, Illinois. Chaddick Institute for Metropolitan Development.

SCHWIETERMAN, J. P., LIVINGSTON, M. \& VAN DER SLOT, S. 2018. Partners in transit: A review of partnerships between transportation network companies and public agencies in the United States. Chaddick Institute for Metropolitan Development.

SHIRGAOKAR, M. 2020. Expanding seniors' mobility through phone apps: Potential responses from the private and public sectors. Journal of Planning Education and Research, 40, 405-415. 
TAYLOR, B. \& MORRIS, E. A. 2015. Public transportation objectives and rider demographics: are transit's priorities poor public policy? Transportation, 42, 347367.

TOMS, K. \& SONG, W. 2016. Spatial Analysis of the Relationship Between Levels of Service Provided by Public Transit and Areas of High Demand in Jefferson County, Kentucky. Papers in Applied Geography, 2, 147-159.

WELCH, T. F. \& MISHRA, S. 2013. A measure of equity for public transit connectivity. Journal of Transport Geography, 33, 29-41.

WELLS, K. \& THILL, J.-C. 2012. Do transit-dependent neighborhoods receive inferior bus access? A neighborhood analysis in four US cities. Journal of Urban Affairs, 34, 4363.

WIDENER, M. J., FARBER, S., NEUTENS, T. \& HORNER, M. 2015. Spatiotemporal accessibility to supermarkets using public transit: an interaction potential approach in Cincinnati, Ohio. Journal of Transport Geography, 42, 72-83.

YANG, T. C., NOAH, A. J. \& SHOFF, C. 2015. Exploring geographic variation in US mortality rates using a spatial Durbin approach. Population, space and place, 21, 1837.

YOUNG, M., ALLEN, J. \& FARBER, S. 2020. Measuring when Uber behaves as a substitute or supplement to transit: An examination of travel-time differences in Toronto. Journal of Transport Geography, 82, 102629. 


\section{Appendix A: Ride-hailing Model Coefficients and Additional Effects}

\section{TABLE A.1 Ride-hailing model coefficients}

\begin{tabular}{|c|c|c|c|c|c|c|c|c|c|c|c|c|}
\hline & \multicolumn{2}{|c|}{ Pickups (all) } & \multicolumn{2}{|c|}{ Dropoffs (all) } & \multicolumn{2}{|c|}{$\begin{array}{c}\text { Pickups } \\
\text { (weekday night) }\end{array}$} & \multicolumn{2}{|c|}{$\begin{array}{c}\text { Dropoffs } \\
\text { (weekday night) }\end{array}$} & \multicolumn{2}{|c|}{$\begin{array}{c}\text { Pickups } \\
\text { (weekend night) }\end{array}$} & \multicolumn{2}{|c|}{$\begin{array}{c}\text { Dropoffs } \\
\text { (weekend night) }\end{array}$} \\
\hline & Estimate & $\begin{array}{l}\text { Lagged } \\
\text { estimate }\end{array}$ & Estimate & $\begin{array}{l}\text { Lagged } \\
\text { estimate }\end{array}$ & Estimate & $\begin{array}{l}\text { Lagged } \\
\text { estimate }\end{array}$ & Estimate & $\begin{array}{l}\text { Lagged } \\
\text { estimate }\end{array}$ & Estimate & $\begin{array}{l}\text { Lagged } \\
\text { estimate }\end{array}$ & Estimate & $\begin{array}{l}\text { Lagged } \\
\text { estimate }\end{array}$ \\
\hline \multirow[t]{2}{*}{ (Intercept) } & -7.44 & & -6.80 & & -7.63 & & -6.40 & & -6.19 & & -3.86 & \\
\hline & $(4.03)$ & & $(4.01)$ & & $(4.88)$ & & $(4.56)$ & & $(4.66)$ & & $(4.17)$ & \\
\hline \multicolumn{13}{|l|}{ Demographic characteristics } \\
\hline \multirow[t]{2}{*}{ Black population (\%) } & -0.00 & $0.02^{* *}$ & -0.00 & $0.02^{* *}$ & -0.01 & 0.01 & -0.01 & $0.01^{*}$ & $-0.0 I^{*}$ & 0.01 & $-0.0 I^{* *}$ & $0.01^{*}$ \\
\hline & $(0.00)$ & $(0.01)$ & $(0.00)$ & $(0.01)$ & $(0.00)$ & $(0.01)$ & $(0.00)$ & $(0.0 \mathrm{I})$ & $(0.00)$ & $(0.0 \mathrm{I})$ & $(0.00)$ & $(0.01)$ \\
\hline \multirow[t]{2}{*}{ Latino population (\%) } & -0.01 & $0.02^{*}$ & $-0.0 I^{*}$ & $0.02^{* *}$ & $-0.01^{*}$ & $0.02^{*}$ & -0.00 & 0.01 & -0.01 & 0.01 & -0.01 & 0.01 \\
\hline & $(0.00)$ & $(0.01)$ & $(0.00)$ & $(0.01)$ & $(0.01)$ & $(0.01)$ & $(0.01)$ & $(0.01)$ & $(0.01)$ & $(0.01)$ & $(0.01)$ & $(0.01)$ \\
\hline \multirow[t]{2}{*}{ Asian population (\%) } & 0.00 & 0.02 & -0.00 & $0.03^{*}$ & -0.01 & 0.03 & 0.00 & 0.02 & -0.00 & $0.03^{*}$ & 0.00 & 0.02 \\
\hline & $(0.01)$ & $(0.01)$ & $(0.01)$ & $(0.01)$ & $(0.01)$ & $(0.01)$ & $(0.01)$ & $(0.01)$ & $(0.01)$ & $(0.01)$ & $(0.01)$ & $(0.01)$ \\
\hline \multirow[t]{2}{*}{ Foreign-born population (\%) } & -0.00 & 0.01 & -0.00 & 0.01 & -0.01 & -0.00 & -0.01 & 0.01 & -0.01 & -0.01 & -0.01 & 0.00 \\
\hline & $(0.01)$ & $(0.01)$ & $(0.01)$ & $(0.01)$ & $(0.01)$ & $(0.01)$ & $(0.01)$ & $(0.01)$ & $(0.01)$ & $(0.01)$ & $(0.01)$ & $(0.01)$ \\
\hline \multicolumn{13}{|l|}{ Transit dependency } \\
\hline \multirow[t]{2}{*}{ No household vehicles (\%) } & $0.0 I^{* * *}$ & -0.00 & $0.01^{* * *}$ & -0.00 & $0.02^{* * *}$ & -0.00 & $0.01^{* * * *}$ & -0.00 & $0.02^{* * * *}$ & -0.01 & $0.01^{* * * *}$ & -0.01 \\
\hline & $(0.00)$ & $(0.01)$ & $(0.00)$ & $(0.01)$ & $(0.00)$ & $(0.01)$ & $(0.00)$ & $(0.01)$ & $(0.00)$ & $(0.01)$ & $(0.00)$ & $(0.01)$ \\
\hline \multirow[t]{2}{*}{ Population age 10-17 (\%) } & -0.01 & $-0.06^{*}$ & -0.01 & $-0.06^{*}$ & -0.02 & $-0.08^{*}$ & -0.02 & $-0.07^{*}$ & -0.02 & $-0.06^{*}$ & -0.02 & $-0.06^{*}$ \\
\hline & $(0.01)$ & $(0.03)$ & $(0.01)$ & $(0.03)$ & $(0.01)$ & $(0.03)$ & $(0.01)$ & $(0.03)$ & $(0.01)$ & $(0.03)$ & $(0.01)$ & $(0.03)$ \\
\hline \multirow[t]{2}{*}{ Population age $65+(\%)$} & -0.01 & $-0.04^{*}$ & -0.01 & $-0.03^{*}$ & $-0.02^{*}$ & -0.04 & $-0.02^{* *}$ & -0.02 & $-0.02^{*}$ & $-0.04^{*}$ & $-0.02^{*}$ & -0.02 \\
\hline & $(0.01)$ & $(0.02)$ & $(0.01)$ & $(0.02)$ & $(0.01)$ & $(0.02)$ & $(0.01)$ & $(0.02)$ & $(0.01)$ & $(0.02)$ & $(0.01)$ & $(0.02)$ \\
\hline \multirow[t]{2}{*}{ Unemployed (\%) } & $-0.03^{* *}$ & 0.02 & $-0.03^{* * k}$ & 0.02 & $-0.04^{* * * *}$ & 0.05 & $-0.05^{* * * *}$ & 0.02 & $-0.04^{* * *}$ & 0.02 & $-0.04^{* * *}$ & 0.01 \\
\hline & $(0.01)$ & $(0.02)$ & $(0.01)$ & $(0.02)$ & $(0.01)$ & $(0.03)$ & $(0.01)$ & $(0.02)$ & $(0.01)$ & $(0.03)$ & $(0.01)$ & $(0.02)$ \\
\hline \multirow[t]{2}{*}{ Median household income (log \$) } & $0.36^{*}$ & 0.49 & $0.32^{*}$ & 0.48 & 0.20 & 0.54 & 0.20 & 0.38 & 0.28 & 0.39 & 0.21 & 0.20 \\
\hline & $(0.15)$ & $(0.34)$ & $(0.15)$ & $(0.34)$ & $(0.19)$ & $(0.4 I)$ & $(0.17)$ & $(0.38)$ & $(0.18)$ & $(0.39)$ & $(0.16)$ & $(0.35)$ \\
\hline
\end{tabular}




\begin{tabular}{|c|c|c|c|c|c|c|c|c|c|c|c|c|}
\hline \multirow[b]{2}{*}{ Population density (log) } & \multicolumn{2}{|c|}{ Pickups (all) } & \multicolumn{2}{|c|}{ Dropoffs (all) } & \multicolumn{2}{|c|}{$\begin{array}{c}\text { Pickups } \\
\text { (weekday night) }\end{array}$} & \multicolumn{2}{|c|}{$\begin{array}{c}\text { Dropoffs } \\
\text { (weekday night) }\end{array}$} & \multicolumn{2}{|c|}{$\begin{array}{c}\text { Pickups } \\
\text { (weekend night) }\end{array}$} & \multicolumn{2}{|c|}{$\begin{array}{c}\text { Dropoffs } \\
\text { (weekend night) }\end{array}$} \\
\hline & 0.12 & -0.05 & -0.01 & 0.06 & 0.02 & 0.05 & $0.49^{* * * *}$ & -0.19 & 0.15 & -0.05 & $0.50^{* * * *}$ & -0.20 \\
\hline & $(0.07)$ & $(0.16)$ & $(0.07)$ & $(0.16)$ & $(0.08)$ & $(0.19)$ & $(0.08)$ & $(0.19)$ & $(0.08)$ & $(0.19)$ & $(0.07)$ & $(0.17)$ \\
\hline \multirow[t]{2}{*}{ Low-wage empl. density (log) } & $0.16^{* * *}$ & 0.09 & $0.18^{* * *}$ & 0.10 & $0.22^{* * *}$ & 0.24 & 0.04 & 0.16 & $0.20^{* * *}$ & 0.19 & 0.04 & 0.11 \\
\hline & $(0.04)$ & $(0.13)$ & $(0.04)$ & $(0.13)$ & $(0.05)$ & $(0.16)$ & $(0.04)$ & $(0.14)$ & $(0.05)$ & $(0.15)$ & $(0.04)$ & $(0.13)$ \\
\hline \multirow[t]{2}{*}{ High-wage empl. density (log) } & 0.05 & -0.02 & $0.06^{*}$ & -0.03 & 0.04 & -0.11 & 0.05 & -0.05 & 0.01 & -0.10 & 0.02 & -0.02 \\
\hline & $(0.03)$ & $(0.09)$ & $(0.03)$ & $(0.09)$ & $(0.04)$ & $(0.11)$ & $(0.04)$ & $(0.10)$ & $(0.04)$ & $(0.11)$ & $(0.03)$ & $(0.10)$ \\
\hline \multicolumn{13}{|l|}{ Transit characteristics } \\
\hline \multirow[t]{2}{*}{ Transit stop density $\left(\mathrm{I} / \mathrm{km}^{2}\right)$} & $-0.20^{*}$ & 0.27 & $-0.23^{* *}$ & 0.31 & -0.14 & 0.46 & $-0.27^{* *}$ & 0.31 & -0.12 & $0.68^{*}$ & $-0.25^{* *}$ & $0.52^{*}$ \\
\hline & $(0.09)$ & $(0.23)$ & $(0.09)$ & $(0.23)$ & $(0.11)$ & $(0.28)$ & $(0.10)$ & $(0.26)$ & $(0.10)$ & $(0.27)$ & $(0.09)$ & $(0.24)$ \\
\hline \multirow[t]{2}{*}{ Transit route density $\left(1 / \mathrm{km}^{2}\right)$} & -0.07 & 0.22 & 0.02 & 0.09 & 0.05 & -0.30 & -0.09 & 0.02 & -0.04 & -0.31 & -0.08 & -0.13 \\
\hline & $(0.08)$ & $(0.20)$ & $(0.08)$ & $(0.20)$ & $(0.10)$ & $(0.24)$ & $(0.10)$ & $(0.23)$ & $(0.10)$ & $(0.23)$ & $(0.09)$ & $(0.2 I)$ \\
\hline \multirow[t]{2}{*}{ Median transit headway ( $\min )$} & -0.00 & 0.00 & -0.00 & 0.00 & -0.00 & 0.00 & -0.00 & 0.00 & -0.00 & -0.00 & $-0.00^{*}$ & -0.00 \\
\hline & $(0.00)$ & $(0.00)$ & $(0.00)$ & $(0.00)$ & $(0.00)$ & $(0.00)$ & $(0.00)$ & $(0.00)$ & $(0.00)$ & $(0.00)$ & $(0.00)$ & $(0.00)$ \\
\hline \multirow[t]{2}{*}{ Number overnight stops } & $0.00^{* * * *}$ & $-0.00^{\text {*** }}$ & $0.00^{* * *}$ & $-0.00^{* * * * *}$ & $0.00^{* * *}$ & $-0.00^{* *}$ & $0.00^{* * * *}$ & $-0.00^{* * *}$ & $0.00^{* * * k}$ & $-0.00^{* * *}$ & $0.00^{* * * * k}$ & $-0.00^{* * * *}$ \\
\hline & $(0.00)$ & $(0.00)$ & $(0.00)$ & $(0.00)$ & $(0.00)$ & $(0.00)$ & $(0.00)$ & $(0.00)$ & $(0.00)$ & $(0.00)$ & $(0.00)$ & $(0.00)$ \\
\hline \multirow[t]{2}{*}{ Rail station within $400 \mathrm{~m}$} & $0.19^{*}$ & -0.22 & $0.23^{* *}$ & -0.22 & 0.19 & -0.06 & 0.15 & -0.13 & $0.24^{* *}$ & -0.13 & 0.16 & -0.08 \\
\hline & $(0.08)$ & $(0.17)$ & $(0.08)$ & $(0.17)$ & $(0.10)$ & $(0.21)$ & $(0.09)$ & $(0.19)$ & $(0.09)$ & $(0.20)$ & $(0.08)$ & $(0.18)$ \\
\hline \multirow[t]{2}{*}{ rho } & $0.53 * * *$ & & $0.52^{* * *}$ & & $0.44^{* * *}$ & & $0.48^{* * * *}$ & & $0.45^{* * *}$ & & $0.52^{* * * *}$ & \\
\hline & $(0.05)$ & & $(0.05)$ & & $(0.06)$ & & $(0.05)$ & & $(0.06)$ & & $(0.05)$ & \\
\hline Num. obs. & 800 & & 800 & & 800 & & 800 & & 800 & & 800 & \\
\hline Parameters & 37 & & 37 & & 37 & & 37 & & 37 & & 37 & \\
\hline Log Likelihood & -980.94 & & -980.24 & & -1133.38 & & -1079.28 & & -1098.76 & & -1015.69 & \\
\hline AIC (Linear model) & 2111.28 & & 2105.01 & & 2387.92 & & 2292.57 & & 2323.61 & & 2180.52 & \\
\hline AIC (Spatial model) & 2035.88 & & 2034.48 & & 2340.76 & & 2232.56 & & 2271.52 & & 2105.38 & \\
\hline LR test: statistic & $77.40 * * *$ & & $72.53 * * *$ & & $49.16 * * *$ & & $62.01 * * *$ & & $54.09 * * *$ & & $77.14 * * *$ & \\
\hline Nagelkerke $\mathrm{R}^{2}$ & 0.80 & & 0.80 & & 0.79 & & 0.78 & & 0.81 & & 0.83 & \\
\hline Lagrange Multiplier test & 3.41 & & 2.22 & & 3.75 & & 3.09 & & $8.17^{* *}$ & & $4.74 *$ & \\
\hline
\end{tabular}

${ }^{*} * *$ p $<0.001 ;{ }^{* *} \mathrm{p}<0.01 ;{ }^{*} \mathrm{p}<0.05$

Note: Dependent variables are natural log transformed (all trip ends) or natural log plus one transformed (overnight models). Standard errors in parentheses. 
TABLE A.2 Additional ride-hailing model effects

\begin{tabular}{|c|c|c|c|c|c|c|c|c|c|c|c|c|}
\hline & \multicolumn{3}{|c|}{$\begin{array}{c}\text { Pickups } \\
\text { (weekday night) }\end{array}$} & \multicolumn{3}{|c|}{$\begin{array}{c}\text { Drop-offs } \\
\text { (weekday night) }\end{array}$} & \multicolumn{3}{|c|}{$\begin{array}{c}\text { Pickups } \\
\text { (weekend night) }\end{array}$} & \multicolumn{3}{|c|}{$\begin{array}{c}\text { Dropoffs } \\
\text { (weekend night) }\end{array}$} \\
\hline & Direct & Indirect & Total & Direct & Indirect & Total & Direct & Indirect & Total & Direct & Indirect & Total \\
\hline Black population (\%) & -0.007 & 0.018 & 0.012 & -0.006 & 0.021 & 0.015 & -0.008 & 0.012 & 0.004 & -0.012 & 0.015 & 0.003 \\
\hline Latino population (\%) & -0.012 & 0.021 & 0.009 & -0.004 & 0.013 & 0.009 & -0.006 & 0.018 & 0.012 & -0.007 & 0.013 & 0.006 \\
\hline Asian population (\%) & -0.004 & 0.043 & 0.039 & 0.003 & 0.034 & 0.037 & 0.000 & $0.05 I$ & 0.052 & 0.003 & 0.036 & 0.039 \\
\hline Foreign-born population (\%) & -0.005 & -0.010 & -0.016 & -0.011 & 0.004 & -0.006 & -0.008 & -0.025 & -0.033 & -0.012 & -0.004 & -0.017 \\
\hline No household vehicles (\%) & 0.017 & 0.006 & 0.023 & 0.015 & 0.011 & 0.025 & 0.016 & 0.002 & 0.018 & 0.013 & 0.003 & 0.016 \\
\hline Population age 10-17 (\%) & -0.022 & -0.149 & -0.171 & -0.021 & -0.152 & -0.173 & -0.026 & -0.132 & -0.158 & -0.020 & -0.147 & -0.167 \\
\hline Population age $65+(\%)$ & -0.020 & -0.076 & -0.096 & -0.022 & -0.049 & -0.070 & -0.019 & -0.077 & -0.096 & -0.017 & -0.057 & -0.074 \\
\hline Unemployed (\%) & -0.043 & 0.053 & 0.010 & -0.046 & -0.012 & -0.058 & -0.044 & 0.002 & -0.042 & -0.039 & -0.028 & -0.067 \\
\hline Median household income (log \$) & 0.237 & 1.090 & 1.326 & 0.225 & 0.892 & 1.117 & 0.309 & 0.927 & 1.236 & 0.235 & 0.621 & 0.856 \\
\hline Population density (log) & 0.025 & 0.103 & 0.128 & 0.492 & 0.090 & 0.582 & 0.151 & 0.039 & 0.190 & 0.499 & 0.112 & 0.611 \\
\hline Low-wage employment density (log) & 0.238 & 0.583 & 0.821 & 0.052 & 0.338 & 0.390 & 0.210 & 0.490 & 0.700 & 0.044 & 0.268 & 0.312 \\
\hline High-wage employment density (log) & 0.036 & -0.158 & -0.122 & 0.052 & -0.039 & 0.012 & 0.002 & -0.179 & -0.177 & 0.022 & -0.022 & 0.000 \\
\hline Transit stop density (log) & -0.116 & 0.697 & 0.581 & -0.259 & 0.341 & 0.082 & -0.087 & 1.105 & 1.018 & -0.223 & 0.781 & 0.558 \\
\hline Transit route density $(\log )$ & 0.036 & -0.483 & -0.447 & -0.092 & -0.043 & -0.135 & -0.053 & -0.588 & -0.642 & -0.096 & -0.342 & -0.438 \\
\hline Median transit headway (min) & 0.000 & 0.001 & 0.001 & 0.000 & 0.000 & 0.000 & 0.000 & -0.001 & -0.001 & -0.001 & -0.002 & -0.003 \\
\hline Number overnight stops & 0.002 & -0.001 & 0.001 & 0.002 & -0.001 & 0.001 & 0.002 & -0.001 & 0.001 & 0.002 & -0.002 & 0.001 \\
\hline Rail station within $400 \mathrm{~m}$ & 0.190 & 0.045 & 0.235 & 0.144 & -0.113 & 0.031 & 0.239 & -0.044 & 0.194 & 0.156 & -0.005 & 0.152 \\
\hline
\end{tabular}

Note: Bold indicates $p<0.05$, italics $p<0.10$ 